\title{
Preconception Genetic Counselling in a Filipino Couple with Family History of Trisomy 18
}

\author{
Peter James B. Abad ${ }^{1,2}$ and Mercy Y. Laurino ${ }^{2,3}$ \\ ${ }^{1}$ College of Nursing, University of the Philippines Manila \\ ${ }^{2}$ Department of Pediatrics, College of Medicine and Philippine General Hospital, University of the Philippines Manila \\ ${ }^{3}$ Seattle Care Cancer Alliance, Seattle, WA, USA
}

\begin{abstract}
Preconception genetic counselling offers an opportunity for prospective parents to understand and adjust to the medical, familial, and psychosocial implications of genetic contributions to pregnancy outcomes. In this paper, we will illustrate how preconception genetic counselling made a difference to a Filipino couple with a previous child diagnosed with Trisomy 18.
\end{abstract}

Key Words: preconception counselling, Trisomy 18, genetic counselling, Philippines

\section{Introduction}

Preconception genetic counselling offers prospective parents an opportunity to explore, identify, and address genetic risk factors that may pose pregnancy risks, and to plan pregnancies with appropriate prenatal, intrapartum, and postnatal care. ${ }^{1}$ This case report will illustrate how preconception genetic counselling made a difference in a Filipino couple with a previous pregnancy outcome of a child with Trisomy 18.

\section{Clinical Report}

We saw a couple in clinic who had a previous history of a child with Trisomy 18. The child was born full term via Caesarean Section due to breech presentation. At birth, the child was observed to be of low birthweight at 1.5 kilograms and with fair cry, suck, and activity. Upon physical examination, the child was noted to have low set ears and rocker bottom feet. The child stayed in the hospital for three days for further assessment and observation after which the child was discharged with instructions to have chromosomal analysis done. At two months of age, the child was observed to have recurrent sleep apnea for which consultation at a local private hospital was sought. Upon physical examination, a cardiac murmur was noted for which a 2Dechocardiography was carried out and revealed ventricular septal defect, patent ductus arteriosus and pulmonary

Corresponding author: Peter James B. Abad, MSc, RN College of Nursing

University of the Philippines Manila

Sotejo Hall, Pedro Gil St., Ermita, Manila 1000 Philippines

Telephone: +6325231477

Email: pbabad@up.edu.ph hypertension. The chromosomal analysis that was initially requested showed a result of full trisomy $18(47, \mathrm{XX},+18)$. The baby passed away at three months of age due to cardiorespiratory failure.

The couple reported that this was a planned pregnancy. The consultand had regular prenatal check-up at a private obstetrician-gynaecologist. She denied exposure to cigarette smoke, alcoholic beverage and maternal infection during the pregnancy.

The couple were both college graduates and were employed at the time. The consultand works as an office staff while her husband works in a telecommunications company.

A three-generation family medical history was obtained during the clinic consult (Figure 1).

\section{Discussion}

Trisomy 18 is the second most common autosomal trisomy with a live born prevalence of 1 in $6,000 .^{2}$ Babies born with Trisomy 18 have characteristic features. Most of them also have cardiovascular and gastrointestinal malformations. ${ }^{3}$ About $98 \%$ of conceptus with Trisomy 18 die in fetal life and only about $5-10 \%$ of affected babies live beyond the first year of life. Mortality in babies with Trisomy 18 is commonly due to congenital heart and renal defects. $^{4}$

\section{Family History and Risk Assessment}

Trisomy 18 is due to the presence of extra material from chromosome 18 as a result of either meiotic nondisjunction or an unbalanced chromosomal structural arrangement that involves chromosome 18. Cytogenetically, about $94 \%$ of all cases of Trisomy 18 are categorized as full trisomy, about 5\% are mosaic, and remaining are a case of partial trisomy 18q.5,6 Knowing the cytogenetic type of Trisomy 18 is important to ascertain the recurrence risk in the family. Full trisomy 18 has a recurrence risk of around $1 \%{ }^{4}$ and is associated with advanced maternal age (i.e., women who are 35 years or older at the time of delivery). ${ }^{7}$ Partial trisomy 18 has a higher recurrence risk compared to the full trisomy 18 and this depends on whether a parent is a carrier of a balanced translocation. ${ }^{4}$ In such a case, parents need to undergo karyotyping to rule out the possibility of being carriers of a 


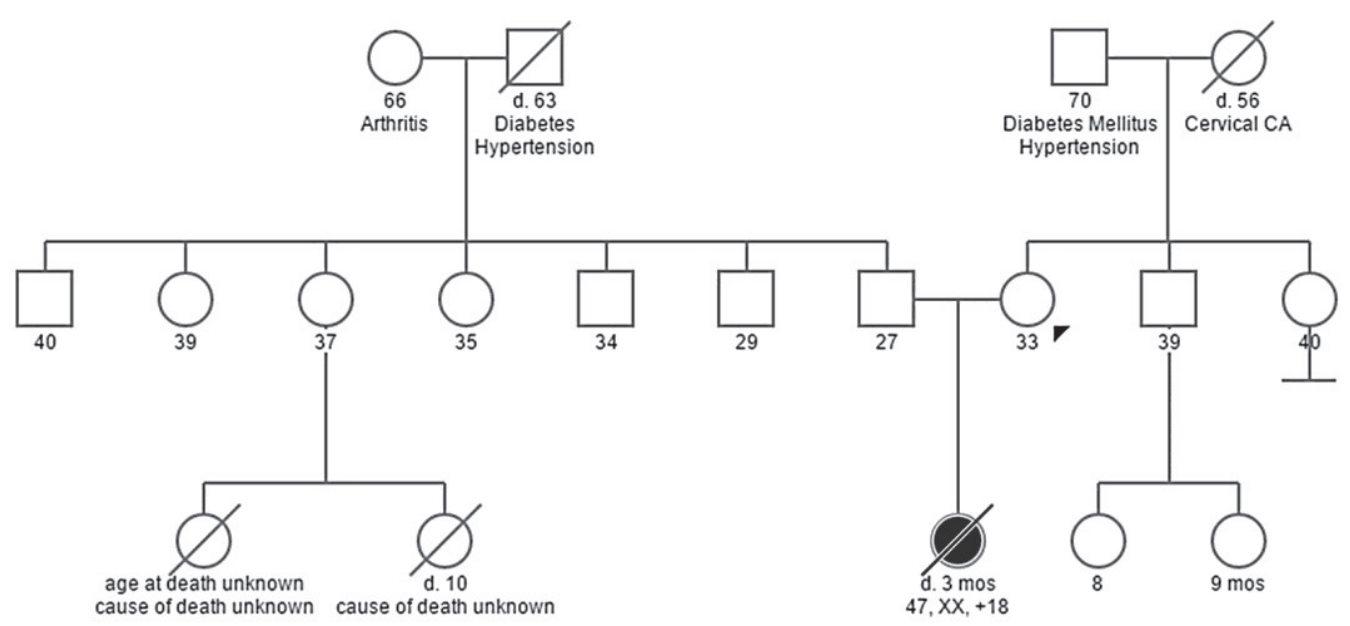

Figure 1. Three-generation family medical history of the couple.

balanced translocation. Chromosomal analysis of parents should be done preconceptionally rather than during pregnancy to allow for the discussion of reproductive planning to occur without any concern of the outcomes of a current pregnancy. ${ }^{8}$

In our case, the Trisomy 18, confirmed cytogenetically as the full trisomy type $(47, X X,+18)$, is not inherited and caused by meiotic nondisjunction. We explained that the chance of having a child with Trisomy 18 is a random occurrence and the chance of having another affected child is related to advancing maternal age. We also discussed the occurrence of other trisomies in relation to advanced maternal age since there are reports in literature of other trisomies occurring within the same family. ${ }^{9}$

\section{Options for prenatal diagnosis}

A number of non-invasive or invasive methods can be done for prenatal screening and diagnosis. Non-invasive methods include maternal serum biochemical screening, measurement of fetal nuchal translucency through ultrasonography, congenital anomaly scan, and fetal echocardiography if congenital heart defects are detected in the congenital anomaly scan. ${ }^{10}$ Invasive methods include chorionic villus sampling and amniocentesis which have inherent risk to the fetus. ${ }^{11}$ While there are advancements in prenatal diagnosis, use of prenatal diagnosis in the Philippines remains to be limited. ${ }^{11}$ Ultrasonography is the most extensively used prenatal diagnostic procedure in the country usually done during the second trimester.

Given the resources in our setting, we presented the option that should the consultand get pregnant again, congenital anomaly scan via an ultrasound can be done to potentially detect malformations suggestive of a chromosomal problem. We underscored that prenatal diagnosis is done to prepare and anticipate for the birth of the child should there be any abnormal finding in the prenatal screening or diagnostic tests. Termination of pregnancy is not allowed legally in the Philippines. Prenatal care and multivitamins, such as folic acid, are also recommended to reduce the risk of other birth defects, specifically neural tube defects.

\section{Psychosocial support}

Both the consultand and her husband expressed their intention to learn more about Trisomy 18. As mentioned earlier, they expressed that they would like to have another child; however, they expressed fear that they may not be 'genetically' compatible and they could have another affected child in the future. Given the questions of the couple and consultand's feelings of guilt and self-blame, we emphasized that the occurrence or recurrence of the condition is not caused by what the couple did or did not do during the prenatal period. We reviewed basic concepts of the cell, chromosomes and the genes and the etiology of trisomy 18 and meiotic nondisjunction. We explained the random nature of having a child with extra chromosome such as trisomy 18 and that most have been associated with the risk of meiotic nondisjunction which increases with maternal age. In view of the principle of non-directiveness and given the amount of information they received during this counselling session, we gave the couple ample time to digest the information provided to them so they can make an informed decision about their situation.

\section{Statement of Authorship}

All authors have approved the final version submitted.

\section{Author Disclosure}

All the authors declared no conflicts of interest.

\section{Funding Source}

None. 


\section{References}

1. Wille MC, Weitz B, Kerper P, Frazier S. Advances in preconception genetic counseling. J Perinat Neonatal Nurs. 2004;18(1):28-40.

2. Matthews AL. Chromosomal abnormalities: Trisomy 18, Trisomy 13, deletions, and microdeletions. J Perinat Neonatal Nurs. 1999; 13(2):59-75.

3. Chen H. Atlas of Genetic Diagnosis and Counseling. Totowa, New Jersey: Humana Press; 2006.

4. Cereda A, Carey JC. The trisomy 18 syndrome. Orphanet J Rare Dis. 2012; 7:81

5. Embleton ND, Wyllie JP, Wright MJ, Burn J, Hunter S. Natural history of trisomy 18. Arch Dis Child Fetal Neonatal Ed. 1996;75(1):F38-41.

6. Wu J, Springett A, Morris JK. Survival of trisomy 18 (Edwards syndrome) and trisomy 13 (Patau Syndrome) in England and Wales: 2004-2011. Am J Med Genet A. 2013; 161A(10):2512-8.
7. Drugan A, Yaron Y, Zamir R, Ebrahim SA, Johnson MP, Evans MI Differential effect of advanced maternal age on prenatal diagnosis of trisomies 13, 18 and 21. Fetal Diagn Ther. 1999;14(3):181-4.

8. Shapira SK, Dolan S. Genetic risks to the mother and the infant: Assessment, counseling, and management. Matern Child Health J. 2006; 10(5 Suppl):S143-6.

9. Baty BJ, Jorde LB, Blackburn BL, Carey JC. Natural History of Trisomy 18 and Trisomy 13: II. Psychomotor Development. Am J Med Genet. 1994; 49(2):189-94.

10. Padilla CD, Cutiongco-dela Paz EM. Genetics and genomic medicine in the Philippines. Mol Genet Genomic Med. 2016; 4(5):494-503.

11. Cutiongco-dela Paz EM. Prenatal diagnosis and its role in reproductive risk screening, prevention and treatment of genetic diseases: Is the Philippines ready for it? Acta Med Philipp. 2006; 40(2):54-7. 